\title{
RIGIDITY FOR COMPLETE WEINGARTEN HYPERSURFACES
}

\author{
M. DAJCZER AND K. TENENBLAT
}

\begin{abstract}
We classify, locally and globally, the ruled Weingarten hypersurfaces of the Euclidean space. As a consequence of the local classification and a rigidity theorem of Dajczer and Gromoll, it follows that a complete Weingarten hypersurface which does not contain an open subset of the form $L^{3} \times \mathbf{R}^{n-3}$, where $L^{3}$ is unbounded and $n \geq 3$, is rigid.
\end{abstract}

\section{INTRODUCTION}

Recently Dajczer and Gromoll [DG $]_{2}$ showed that a complete hypersurface $M^{n}, n \geq 4$, of the euclidean space $\mathbf{R}^{n+1}$ is rigid, unless it contains an open subset $U$ such that either $U=L^{3} \times \mathbf{R}^{n-3}$ with $L^{3}$ unbounded or $U$ is completely ruled. We recall that a completely ruled submanifold is a ruled submanifold with complete rulings. It is not known if there exists a nowhere ruled three-dimensional irreducible hypersurface which is not rigid (see $\left[D_{2}\right]$ ).

We observe that there is an abundance of hypersurfaces of the euclidean space which admit local isometric deformations. A classification of such hypersurfaces was obtained by Sbrana [S] and Cartan [C]. A special case is given by the minimal hypersurfaces of rank two discussed in $[D G]_{1}$.

In this paper we consider the rigidity question for complete hypersurfaces $M^{n}$ which satisfy the additional condition of being Weingarten, i.e. there exists a differentiable function relating the mean curvature and the scalar curvature of $M$. Our main result is the following.

Theorem A. Let $M^{n}, n \geq 4$, be a complete Weingarten immersed hypersurface of $\mathbf{R}^{n+1}$, which does not contain an open subset $U=L^{3} \times \mathbf{R}^{n-3}$ with $L^{3}$ unbounded. Then $M$ is rigid.

The above result is an immediate consequence of the rigidity theorem of Dajczer and Gromoll and the following local classification of ruled Weingarten hypersurfaces.

Theorem B. Let $M^{n}, n \geq 3$, be a connected ruled Weingarten hypersurface of $\mathbf{R}^{n+1}$. Then $M^{n}$ is either

(i) flat;

Received by the editors April 13, 1987.

1980 Mathematics Subject Classification (1985 Revision). Primary 53A07, 53B25, 53C40, 53C42.

The authors were partially supported by CNPq. 
or it is an open subset of one of the following:

(ii) $Q^{3} \times \mathbf{R}^{n-3}$, where $Q^{3} \subset \mathbf{R}^{4}$ is a cone over a product of circles in $S^{3}$, or over a minimal ruled surface in $S^{3}$;

(iii) $Q^{2} \times \mathbf{R}^{n-2}$, where $Q^{2} \subset \mathbf{R}^{3}$ is a ruled helicoidal surface or a hyperboloid of revolution.

The classification for $n=2$ was obtained in 1865 by Beltrami [B] and Dini [D], see (2.29). We observe that the classfication of Theorem B is complete since the minimal ruled surfaces in $S^{3}$ are given in [L], see (2.16).

Now if we assume $M$ to be complete, we have

Corollary C. Let $M^{n}, n \geq 3$, be a complete connected ruled Weingarten hypersurface in $\mathbf{R}^{n+1}$. Then, $M$ is either

(i) a product $Q^{2} \times \mathbf{R}^{n-2}$, where $Q^{2}$ is a complete ruled helicoidal surface of a hyperboloid of revolution; or

(ii) a cylinder over a complete curve.

\section{Preliminares}

Let $M^{n} \subset \mathbf{R}^{n+1}$ be a connected orientable immersed hypersurface endowed with the induced metric. The relative nullity of the immersion at a point $p \in M$, is $\operatorname{ker} A(p)$, where $A$ denotes the second fundamental form of the hypersurface. Suppose that the relative nullity has constant dimension $\bar{\nu}=n-k$. Then the Gauss map $\phi: M^{n} \rightarrow S^{n} \subset \mathbf{R}^{n+1}$ is parallel along each leaf of the relative nullity foliation, and provides (locally) a Gauss parametrization of $M$ as it was defined in $[D G]_{1}$. More precisely, there exists an isometric immersion $g: L^{k} \rightarrow S^{n}$, which is a local parametrization of the image of the Gauss map $\phi$, and a differentiable function $\gamma: L^{k} \rightarrow R$ (support function) such that

$$
\begin{aligned}
X: U & \subset \Lambda \rightarrow M^{n} \subset \mathbf{R}^{n+1}, \\
\quad(x, v) & \mapsto X(x, v)=\gamma(x) g(x)+\operatorname{grad} \gamma(x)+v
\end{aligned}
$$

is a local parametrization of $M^{n}$, where $\Lambda$ is the normal bundle of the immersion $g . X$ is the so-called Gauss parametrization of $M$.

For each $(x, v) \in U \subset \Lambda$, let Hess $\gamma(x)$ denote the hessian of $\gamma$ and $B_{v}$ the second fundamental form of the immersion $g$ at $x \in L^{k}$, relative to the normal vector $v$. Then the selfadjoint operator defined on the tangent space of $L^{k}$ at $x$,

$$
P_{(x, v)}=\gamma(x) I+\operatorname{Hess} \gamma(x)-B_{v}
$$

is nonsingular. Moreover, the second fundamental form $A_{(x, v)}$ of $X$ at $(x, v)$ is given by $-P^{-1}$, when restricted to the orthogonal complement of the relative nullity distribution. We refer to $[D G]_{1}$ for the above results. 
For each vector field $e: L^{k} \rightarrow \mathbf{R}^{n+1}$, we may consider an associated vector field $\bar{e}: U \subset \Lambda \rightarrow \mathbf{R}^{n+1}$ defined by

$$
\bar{e}(x, v)=e(x), \quad \forall(x, v) \in U,
$$

i.e. $\bar{e}$ is the euclidean parallel transport of $e(x)$ along the leaves of the relative nullity foliation of $M$. Therefore, if $e$ is a vector field normal (resp. tangent) to the immersion $g$, then the associated vector field $\bar{e}$ belongs (resp. is orthogonal) to the relative nullity distribution.

In what follows we consider hypersurfaces $M^{n} \subset \mathbf{R}^{n+1}$ with constant index of relative nullity $\bar{\nu}=n-2$, locally parametrized as in (1.1). Moreover, we choose orthonormal vector fields $e_{1}, \ldots, e_{n}$, locally defined on $L^{2}$, such that $e_{1}(x)$, $e_{2}(x)$ are tangent to the immersion $g$ at $x$ and $e_{3}(x), \ldots, e_{n}(x)$ generate the normal space of the immersion in $S^{n}$. Let $\bar{e}_{i}(x, v)=e_{i}(x), 1 \leq i \leq n$, $(x, v) \in U \subset \Lambda$, be the associated vector fields on $M$. With respect to this frame the second fundamental form of $X$ at $(x, v)$ is given by

$$
A=\left(\begin{array}{cc}
-P_{(x, v)}^{-1} & 0 \\
0 & 0
\end{array}\right)
$$

where $P$ is defined by (1.2).

It follows that the mean curvature $\bar{H}$ and the scalar curvature $\bar{S}$ of $M$ at $(x, v)$ are given respectively by

$$
\begin{gathered}
\bar{H}(x, v)=-\operatorname{tr} A=\frac{\operatorname{tr} P}{\operatorname{det} P}, \\
\bar{S}(x, v)=\frac{1}{\operatorname{det} P} .
\end{gathered}
$$

Lemma 1.6. Let $M^{n} \subset \mathbf{R}^{n-1}$ be a ruled immersed hypersurface with constant index of relative nullity $\bar{\nu}=n-2$. Then the immersion $g$ is a ruled surface in $S^{n}$.

Proof. Let

$$
X\left(s, \lambda, \mu_{j}\right)=c(s)+\lambda \xi(s)+\sum_{j=1}^{n-2} \mu_{j} \eta_{j}(s)
$$

be a local parameterization of $M$, where $c(s)$ is a curve orthogonal to the ruling, $\eta_{j}, 1 \leq j \leq n-2$, generate the relative nullity and $\left\{\xi, \eta_{j}\right\}$ generate the ruling of $M^{n}$. Then the Gauss map depends only on the parameters $s, \lambda$, since $\eta_{j}$ generate the relative nullity distribution. Moreover, for $s=s_{0}$, the Gauss map describes a curve which is orthogonal to the subspace generated by $\xi\left(s_{0}\right), \eta_{j}\left(s_{0}\right), 1 \leq j \leq n-2$. Therefore it is contained in a great circle of $S^{n}$. Q.E.D.

Fact 1.7. It follows from the above lemma that if $M$ is a ruled hypersurface then the frame considered earlier may be chosen such that $e_{1}(x)$ is tangent to the ruling of the immersion $g$. Thus the second fundamental form $\theta$ of $g$ with 
values in the normal bundle satisfies $\theta\left(e_{1}, e_{1}\right)=0$. Therefore, the associated frame tangent to $M, \bar{e}_{i}(x, v)=e_{i}(x)$, is such that $\bar{e}_{i}, 3 \leq i \leq n$, generate the relative nullity, $\bar{e}_{i}, 2 \leq i \leq n$, generate the ruling and $\left\langle A \bar{e}_{2}, \bar{e}_{2}\right\rangle=0$.

For such a frame, the second fundamental form of the immersion $g$, with respect to $e_{i}, 3 \leq i \leq n$, will be denoted by

$$
B_{i}(x)=\left(\begin{array}{cc}
0 & \beta_{i} \\
\beta_{i} & \lambda_{i}
\end{array}\right), \quad 3 \leq i \leq n,
$$

and the operator $\gamma(x) I+\operatorname{Hess} \gamma(x)$ will be denoted by

$$
\gamma(x) I+\operatorname{Hess} \gamma(x)=\left(\begin{array}{ll}
0 & h \\
h & \alpha
\end{array}\right) .
$$

Now we assume that the submanifold $M^{n} \subset \mathbf{R}^{n+1}$ is Weingarten, i.e. there exists a differentiable function $F(\bar{H}, \bar{S})=0$. Taking exterior derivatives we obtain

$$
\frac{\partial F}{\partial \bar{H}} d \bar{H}+\frac{\partial F}{\partial \bar{S}} d \bar{S}=0
$$

Therefore, applying to vector fields tangent to $M$, we conclude that

$$
d \bar{H} \wedge d \bar{S}=0,
$$

since the partial derivatives of $F$ are not simultaneously zero.

Fact 1.11. Let $M^{n} \subset \mathbf{R}^{n+1}$ be a ruled Weingarten hypersurface with constant index of relative nullity $\bar{\nu}=n-2$. Then it follows from (1.4) to (1.10) that

$$
d\left(\alpha(x)-\sum_{i=3}^{n} t_{i} \lambda_{i}(x)\right) \wedge d\left(h(x)-\sum_{j=3}^{n} t_{j} \beta_{j}(x)\right)=0
$$

for $t_{i} \in \mathbf{R}$.

\section{Proofs of the theOREMS}

For the proof of Theorem B we will need the following three propositions.

Proposition 2.1. Let $M^{n} \subset \mathbf{R}^{n+1}$ be a connected ruled Weingarten hypersurface without flat points. Suppose that the dimension of the first normal space of $g$ is constant equal to 1 . Then, there exists a totally geodesic submanifold $S^{3} \subset S^{n}$ such that $g\left(L^{2}\right) \subset S^{3}$ is a ruled Weingarten surface which satisfies

$$
H^{2}+c^{2}(K-1)=0,
$$

where $H$ and $K$ are the mean and Gaussian curvature and $c$ is a constant. Moreover, $M^{n}$ is contained in a euclidean product $Q^{3} \times \mathbf{R}^{n-3}$, where $Q^{3} \subset \mathbf{R}^{4}$ is a ruled Weingarten surface with index of relative nullity $\nu=1$.

Proposition 2.2. Let $g: L^{2} \rightarrow S^{3}$ be a connected ruled surface in $S^{3}$ such that

$$
H^{2}+c^{2}(K-1)=0 \text {. }
$$


Then either $H=0$ or $H=c \neq 0$ and $K=0$. In the latter case the immersed surface is contained in the product of two circles.

Proposition 2.3. Let $M^{3} \subset \mathbf{R}^{4}$ be a connected ruled Weingarten hypersurface, with index of relative nullity $\bar{\nu}=1$. Suppose that the image of the Gauss map $g\left(L^{2}\right)$ is either

(i) a minimal surface in $S^{3}$; or

(ii) it is contained in the product of two circles.

Then $M^{3}$ is an open subset of a cone over $g\left(L^{2}\right)$.

We need the following result. Recall that the first normal space of an immersion is the subspace generated by the second fundamental form.

Lemma 2.4. Let $M^{n}$ be a ruled Weingarten hypersurface without flat points. Then, for each $x \in L^{2}$, the dimension of the first normal space $N_{1}$ of $g$ is less than or equal to 1 .

Proof. The ruled hypersurface $M$ has no flat points if and only if the index of relative nullity is constant $\bar{\nu}=n-2$.

Since $M^{n}$ is a ruled hypersurface, it follows from Lemma 1.6 that $g\left(L^{2}\right)$ is a ruled surface. Let $e_{1}(x), e_{2}(x)$ be a locally defined tangent frame to the immersion $g$ such that $e_{1}(x)$ is tangent to the ruling. Let $N_{1}(x)$ be the first normal space of $g$ at $x$. Since $N_{1}$ is generated by $\theta\left(e_{1}, e_{2}\right), \theta\left(e_{2}, e_{2}\right)$, it follows that $\operatorname{dim} N_{1} \leq 2$.

Suppose $\operatorname{dim} N_{1}(x)=2$. We choose $e_{3}(x), e_{4}(x)$ generating $N_{1}$ such that $e_{4}$ is orthogonal to $\theta\left(e_{1}, e_{2}\right)$. Then the second fundamental form with respect to $e_{3}$ and $e_{4}$ in the tangent basis $e_{1}, e_{2}$ is given respectively by

$$
B_{3}=\left(\begin{array}{cc}
0 & \beta_{3} \\
\beta_{3} & \lambda_{3}
\end{array}\right), \quad B_{4}=\left(\begin{array}{cc}
0 & 0 \\
0 & \lambda_{4}
\end{array}\right) \text {. }
$$

Since $M^{n}$ is a Weingarten hypersurface it follows from (1.13) that $\lambda_{4} \beta_{3}=0$. If $\lambda_{4}=0$, then $B_{4}=0$. If $\beta_{3}=0$, then $\theta\left(e_{1}, e_{2}\right)=0$. In both cases we have a contradiction, since we assumed that $\operatorname{dim} N_{1}=2$. Q.E.D.

Proof of Proposition 2.1. Since $M$ is a ruled hypersurface without flat points, the index of relative nullity is constant $\bar{\nu}=n-2$. Let $e_{1}(x), \ldots, e_{n}(x)$ be a orthonormal frame defined locally on $L^{2}$ as in Fact 1.7. Moreover, we can choose $e_{3}(x)$ to generate the first normal space $N_{1}$ of $g$. For such a frame, the second fundamental form of $g(1.8)$ reduces to

$$
B_{3}=\left(\begin{array}{cc}
0 & \beta \\
\beta & \lambda
\end{array}\right), \quad B_{i}=0,4 \leq i \leq n .
$$

Since $M$ is Weingarten, it follows from (1.12) that

$$
d(\alpha-t \lambda) \wedge d(h-t \beta)=0, \quad t \in \mathbf{R} .
$$

Applying (2.6) to the pair $\left(e_{i}, \partial / \partial t\right), i=1,2$, we obtain

$$
-\beta d \alpha+\lambda d h=0 \text {, }
$$




$$
\beta d \lambda-\lambda d \beta=0 .
$$

It follows from (2.8) that there exist constants $c_{1}, c_{2}$, not simultaneously zero, such that

$$
c_{1} \beta+c_{2} \lambda=0
$$

Observe that $c_{2} \neq 0$. In fact if $c_{2}=0$, then from (2.9) we have $\beta=0$. Now, $\operatorname{dim} N_{1}=1$ implies that $\lambda \neq 0$ and (2.7) implies that $h$ is a constant. Therefore, it follows from (1.5) that $\bar{S}=-1 / h^{2}$ is constant. However, Theorem 3.4 in [DG] $]_{1}$ implies that $\operatorname{dim} N_{1}=0$, which is a contradiction.

Therefore, we have

$$
\lambda=c \beta
$$

and $\beta \neq 0$ in $L^{2}$. Moreover, it follows from (2.7) that $\alpha=c h+\bar{c}$, where $c$ and $\bar{c}$ are constants.

Now we prove that the first normal space of the immersion $g: L^{2} \rightarrow S^{n}$ is parallel. In fact, let $\eta$ be any vector field generated by $e_{4}, \ldots, e_{n}$. Then it follows from the Codazzi equation that

$$
B_{\nabla_{e_{1}}^{\frac{1}{1}} \eta} e_{2}=B_{\nabla_{e_{2}}^{\frac{1}{2}} \eta} e_{1},
$$

where $\nabla^{\perp}$ is the connection in the normal bundle. Hence

$$
\left\langle\nabla_{e_{1}}^{\perp} \eta, e_{3}\right\rangle B_{3} e_{2}=\left\langle\nabla_{e_{2}}^{\perp} \eta, e_{3}\right\rangle B_{3} e_{1} .
$$

Using (2.5) we get

$$
\left\langle\nabla_{e_{1}}^{\perp} \eta, e_{3}\right\rangle \beta e_{1}+\left[\left\langle\nabla_{e_{1}}^{\perp} \eta, e_{3}\right\rangle \lambda-\left\langle\nabla_{e_{2}}^{\perp} \eta, e_{3}\right\rangle \beta\right] e_{2}=0 .
$$

Since $\beta \neq 0$ we conclude that

$$
\left\langle\nabla_{e_{1}}^{\perp} \eta, e_{3}\right\rangle=\left\langle\nabla_{e_{2}}^{\perp} \eta, e_{3}\right\rangle=0 .
$$

Hence the first normal space of the immersion $g$ is parallel. It follows that there exists a totally geodesic submanifold $S^{3} \subset S^{n}$ which contains the image of $g$. Therefore, the normal bundle $\Lambda$ of $g$ splits into $\Lambda=\Lambda_{1}+\Lambda_{n-3}$, where $\Lambda_{1}$ is the normal bundle in $S^{3}$ and the orthogonal complement $\Lambda_{n-3}$ is parallel in $\mathbf{R}^{n+1}$. Hence, $M^{n}$ splits as a consequence of the Gauss parametrization.

Finally, from (2.5) we obtain that the mean curvature $H$ and the Gaussian curvature $K$ satisfies $H=\lambda$ and $K-1=\beta^{2}$. Therefore, it follows from (2.10) that $H^{2}+c^{2}(K-1)=0$. Q.E.D.

Fact 2.11. It follows from the preceding proof that if $M^{n}$ satisfies the hypothesis of Proposition 1 then there is a frame locally defined on $L^{2}$ for which

$$
\begin{gathered}
B_{3}=\left(\begin{array}{cc}
0 & \beta \\
\beta & \lambda
\end{array}\right), \quad B_{i}=0,4 \leq i \leq n, \\
\gamma I+\text { Hess } \gamma=\left(\begin{array}{ll}
0 & h \\
h & \alpha
\end{array}\right),
\end{gathered}
$$

where $\lambda=c \beta, \alpha=c h+\bar{c}$, and $c, \bar{c}$ are constants. 
Proof of Proposition 2.2. If the constant $c$ is zero, then $H=0$. Otherwise, we will show that $K=0$ and hence $H=c$.

Let $g: L^{2} \rightarrow S^{3}$ be a parametrized ruled surface in $S^{3}$. We may consider

$$
g(s, t)=\cos t \sigma(s)+\sin t e(s),
$$

where $\sigma(s)$ and $e(s)$ are vectors in $\mathbf{R}^{4}$ such that

$$
|\sigma|=1=|e|, \quad\langle e, \sigma\rangle=0, \quad\left\langle e^{\prime}, \sigma^{\prime}\right\rangle=0 .
$$

Moreover, we may choose the parameter $s$ such that $\left|e^{\prime}\right|=1$. We introduce the following notation

$$
\begin{aligned}
p(s) & =\left\langle e^{\prime}, \sigma^{\prime} \times e \times \sigma\right\rangle=\left(e^{\prime} \sigma^{\prime} e \sigma\right), \\
A(s) & =\left|\sigma^{\prime}\right|^{2}-\left\langle\sigma^{\prime}, e\right\rangle^{2} \\
B(s) & =1-\left\langle\sigma^{\prime}, e\right\rangle^{2} \\
G(s, t) & =A \cos ^{2} t+B \sin ^{2} t
\end{aligned}
$$

We observe that $p=\sqrt{A B}$. Moreover, it follows by a straightforward computation that the mean and Gaussian curvature of the surface are given by

$$
H=\frac{l-2 p\left\langle\sigma^{\prime}, e\right\rangle}{2 G^{3 / 2}}, \quad K-1=-\frac{p^{2}}{G^{2}},
$$

where

$$
\begin{aligned}
l(s, t)= & \cos ^{2} t\left(\sigma^{\prime \prime} \sigma^{\prime} e \sigma\right)+\sin ^{2} t\left(e^{\prime \prime} e^{\prime} e \sigma\right) \\
& +\sin t \cos t\left[\left(\sigma^{\prime \prime} e^{\prime} e \sigma\right)_{+}\left(e^{\prime \prime} \sigma^{\prime} e \sigma\right)\right] .
\end{aligned}
$$

By hypothesis $H^{2}+c^{2}(K-1)=0$, therefore, without loss of generality, we have

$$
l-2 p\left\langle\sigma^{\prime}, e\right\rangle-2 c p G^{1 / 2}=0 .
$$

Taking a derivative with respect to $t$ we get

$$
\partial l / \partial t-c p G^{-1 / 2} \partial G / \partial t=0 .
$$

In particular for $t=0$, it follows from (2.14) and (2.15) that

$$
\left(\sigma^{\prime \prime} e^{\prime} e \sigma\right)+\left(e^{\prime \prime} \sigma^{\prime} e \sigma\right)=0
$$

Hence (2.15) reduces to

$$
2 \sin t \cos t\left[-\left(\sigma^{\prime \prime} \sigma^{\prime} e \sigma\right)+\left(e^{\prime \prime} e^{\prime} e \sigma\right)\right]-c p G^{-1 / 2} \partial G / \partial t=0,
$$

which is equivalent to

$$
\left[\frac{1}{A B}\left(\frac{d}{d s}\left(\sigma^{\prime} \times e \times \sigma\right) e^{\prime} e \sigma\right)-c G^{1 / 2}\right] \frac{\partial G}{\partial t}=0, \quad \forall s, t .
$$

Since $c$ is a nonzero constant, it follows that

$$
\partial G / \partial t=0, \cdot \forall s, t .
$$


Therefore, using (2.12) we get $A=B, p=A=G$. From (2.13) we get $K=0$ and hence $H=c$.

In order to show that the surface is contained in a product of two circles, we consider a local orthonormal frame field such that the second fundamental form is given by

$$
B=\left(\begin{array}{ll}
0 & \beta \\
\beta & \lambda
\end{array}\right)
$$

From $K=0$ and $H=c$, we have $\operatorname{det} B=-1$ and $\lambda=2 c$, so that

$$
B=\left(\begin{array}{cc}
0 & 1 \\
1 & 2 c
\end{array}\right) \text {. }
$$

We conclude the proof by using the uniqueness part of the fundamental theorem for surfaces in the sphere, see [S]. Q.E.D.

Proof of Proposition 2.3. Part (i). Since $\nu=1$, there exists an immersion

$$
g: L^{2} \rightarrow G \subset S^{3}
$$

and a local Gauss parametrization of $M^{3}$ given by

$$
X: \Lambda \rightarrow M^{3} \subset R^{4}, \quad(x, v) \mapsto \gamma(x) g(x)+\operatorname{grad} \gamma(x)+v,
$$

where $\Lambda$ is the normal bundle of the immersion $g$ and $\gamma: L^{2} \rightarrow R$ is a differentiable function.

$M^{3}$ is a ruled hypersurface, therefore it follows from Lemma 1.6 that $g\left(L^{2}\right)$ is a ruled surface in $S^{3}$. Since $g\left(L^{2}\right)$ is also minimal, we have $g$ locally given by

$$
\begin{aligned}
g\left(x_{1}, x_{2}\right)= & \cos x_{1}\left(\cos k x_{2}, \sin k x_{2}, 0,0\right) \\
& +\sin x_{1}\left(0,0, \cos x_{2}, \sin x_{2}\right),
\end{aligned}
$$

where $k$ is a positive constant, see [L or BDJ]. Let us consider the orthonormal tangent frame

$$
e_{1}=\frac{\partial g}{\partial x_{1}}, \quad e_{2}=\frac{1}{\sqrt{E}} \frac{\partial g}{\partial x_{2}},
$$

where $E=k^{2} \cos ^{2} x_{1}+\sin ^{2} x_{1}$. Let $e_{3}$ be a unitary normal vector field for the immersion $g$. Then the second fundamental form with respect to this frame is given by

$$
B(x)=\left(\begin{array}{cc}
0 & -k / E \\
-k / E & 0
\end{array}\right), \quad x=\left(x_{1}, x_{2}\right) .
$$

Let $\bar{e}_{i}$ be the associated frame defined on $M^{3}$, i.e.

$$
\bar{e}_{i}(x, v)=e_{i}(x), \quad 1 \leq i \leq 3,(x, v) \in \Lambda .
$$

Then the second fundamental form for $M^{3} \subset R^{4}$, with respect to this frame, is given by

$$
A(x, v)=\left(\begin{array}{cc}
-P_{(x, v)}^{-1} & 0 \\
0 & 0
\end{array}\right), \quad(x, v) \in \Lambda
$$


where

$$
P(x, v)=\gamma(x)+\text { Hess } \gamma(x)-\left\langle v, e_{3}\right\rangle B(x) .
$$

Moreover, it follows from Fact 2.11 that $\gamma I+$ Hess $\gamma$ is of the form

$$
\gamma I+\operatorname{Hess} \gamma=\left(\begin{array}{ll}
0 & h \\
h & \alpha
\end{array}\right)
$$

where

$$
0=-c k / E, \quad \alpha=c h+\bar{c} .
$$

Hence, $c=0$ and $\alpha=\bar{c}$.

Now, we want to determine $\gamma: L^{2} \rightarrow R$ such that

$$
\gamma I+\operatorname{Hess} \gamma=\left(\begin{array}{ll}
0 & * \\
* & \bar{c}
\end{array}\right)
$$

It follows from (2.17) and (2.19) that $\gamma$ must satisfy

$$
\gamma+\frac{2}{x_{1}^{2}}=0, \quad \gamma+\frac{1}{E}\left(\frac{\partial^{2} \gamma}{\partial x_{2}^{2}}+\sin x_{1} \cos x_{1}\left(1-k^{2}\right) \frac{\partial \gamma}{\partial x_{1}}\right)=\bar{c} .
$$

From the first equation we get

$$
\gamma=f\left(x_{2}\right) \cos x_{1}+h\left(x_{2}\right) \sin x_{1} .
$$

Substituting into (2.20) we get $\bar{c}=0$. Therefore, the trace of $P$ and hence the trace of $A$ is zero, i.e. $M^{3}$ is a minimal surface in $R^{4}$.

To conclude the proof in this case we use [BDJ].

Part (ii). By hypothesis $g\left(L^{2}\right)$ is contained in the product of two circles, therefore the immersion $g$ is locally given by

$$
g\left(x_{1}, x_{2}\right)=r_{1}\left(\sin \frac{x_{1}}{r_{1}}, \cos \frac{x_{1}}{r_{1}}, 0,0\right)+r_{2}\left(0,0, \sin \frac{x_{2}}{r_{2}}, \cos \frac{x_{2}}{r_{2}}\right)
$$

where $r_{1}^{2}+r_{2}^{2}=1$.

Let us consider the orthonormal frame field defined by

$$
e_{1}=r_{1} \frac{\partial g}{\partial x_{1}}-r_{2} \frac{\partial g}{\partial x_{2}}, \quad e_{2}=r_{2} \frac{\partial g}{\partial x_{1}}+r_{1} \frac{\partial g}{\partial x_{2}} \text {. }
$$

Then the second fundamental form of the immersion $g$ with respect to $e_{1}, e_{2}$ is given by

$$
B=\left(\begin{array}{cc}
0 & 1 \\
1 & \frac{r_{2}^{2}-r_{1}^{2}}{r_{1} r_{2}}
\end{array}\right) \text {. }
$$

Let $e_{3}$ be a unitary normal vector field for the immersion $g$ and

$$
\bar{e}_{i}(x, v)=e_{i}(x), \quad 1 \leq i \leq 3,(x, v) \in \Lambda,
$$

the associated frame defined on $M^{3}$. Then the second fundamental form for $M^{3} \subset R^{4}$, with respect to this frame, is given by

$$
A(x, v)=\left(\begin{array}{cc}
-P_{(x, v)}^{-1} & 0 \\
0 & 0
\end{array}\right)
$$

where $P(x, v)=\gamma(x)+\operatorname{Hess} \gamma(x)-\left\langle v, e_{3}\right\rangle B$. 
Moreover, it follows from Fact 2.11 that with respect to this frame

$$
\gamma I+\operatorname{Hess} \gamma=\left(\begin{array}{ll}
0 & h \\
h & \alpha
\end{array}\right)
$$

where

$$
c=\frac{r_{2}^{2}-r_{1}^{2}}{r_{1} r_{2}}, \quad \alpha=c h+\bar{c} .
$$

We want to determine $\gamma: L^{2} \rightarrow \mathbf{R}$ which satisfies the above conditions. It follows from (2.22) that $\gamma$ must satisfy

$$
\begin{gathered}
\gamma+r_{1}^{2} \frac{\partial^{2} \gamma}{\partial x_{1}^{2}}-2 r_{1} r_{2} \frac{\partial^{2} \gamma}{\partial x_{1} \partial x_{2}}+r_{2}^{2} \frac{\partial^{2} \gamma}{\partial x_{2}^{2}}=0, \\
\gamma+r_{1}^{2} \frac{\partial^{2} \gamma}{\partial x_{1}^{2}}+\frac{r_{2}^{4}+r_{1}^{4}}{r_{1} r_{2}} \frac{\partial^{2} \gamma}{\partial x_{1} \partial x_{2}}+r_{2}^{2} \frac{\partial^{2} \gamma}{\partial x_{2}^{2}}=\bar{c} .
\end{gathered}
$$

Subtracting the above equation we get

Therefore

$$
\frac{1}{r_{1} r_{2}} \frac{\partial^{2} \gamma}{\partial x_{1} \partial x_{2}}=\bar{c}
$$

$$
\gamma\left(x_{1}, x_{2}\right)=\bar{c} r_{1} r_{2} x_{1} x_{2}+\gamma_{1}\left(x_{1}\right)+\gamma_{2}\left(x_{2}\right)
$$

where $\gamma_{1}$ and $\gamma_{2}$ are functions which depend only on $x_{1}$ and $x_{2}$ respectively. Substituting (2.24) into (2.23), we obtain

$$
\gamma_{1}+r_{1}^{2} \frac{d^{2} \gamma_{1}}{d x_{1}^{2}}+\gamma_{2}+r_{2}^{2} \frac{d^{2} \gamma_{2}}{d x_{2}^{2}}+\bar{c} r_{1} r_{2} x_{1} x_{2}=2 r_{1}^{2} r_{2}^{2} \bar{c}
$$

Taking derivatives with respect to $x_{1}$, and then with respect to $x_{2}$ we conclude that $\bar{c}=0$. Therefore, (2.24) reduces to

$$
\gamma\left(x_{1}, x_{2}\right)=\gamma_{1}\left(x_{1}\right)+\gamma_{2}\left(x_{2}\right),
$$

where $\gamma_{1}$ and $\gamma_{2}$ satisfy the following equations

$$
\gamma_{1}+r_{1}^{2} \frac{\partial^{2} \gamma_{1}}{\partial x_{1}^{2}}=a, \quad \gamma_{2}+r_{2} \frac{\partial^{2} \gamma_{2}}{\partial x_{2}^{2}}=a
$$

where $a$ is a constant.

Now we want to show that the Gauss parametrization of $M^{3}$ describes a cone over $G$. In fact

where

$$
X\left(x_{1}, x_{2}, s\right)=\gamma\left(r_{1} u_{1}+r_{2} v_{1}\right)+\frac{d \gamma_{1}}{\partial x_{1}} v_{1}+\frac{d \gamma_{2}}{\partial x_{2}} v_{2}+s\left(-r_{2} u_{1}+r_{1} u_{2}\right)
$$

$$
\begin{aligned}
& u_{1}=\left(\sin \frac{x_{1}}{r_{1}}, \cos \frac{x_{1}}{r_{1}}, 0,0\right), \\
& u_{2}=\left(0,0, \sin \frac{x_{2}}{r_{2}}, \cos \frac{x_{2}}{r_{2}}\right), \\
& v_{1}=\partial X / \partial x_{1}, \quad v_{2}=\partial X / \partial x_{2} .
\end{aligned}
$$


It follows from (2.26) and (2.27) that $X\left(x_{1}, x_{2}, s\left(x_{1}, x_{2}\right)\right)$ is constant for

$$
s\left(x_{1}, x_{2}\right)=\frac{a}{r_{1} r_{2}}-\frac{r_{2}}{r_{1}} \gamma_{1}+\frac{r_{1}}{r_{2}} \gamma_{2} \text {, }
$$

which concludes the proof of case (ii). Q.E.D.

Finally, we prove Theorem $B$ using the preceding results.

Proof of Theorem B. Let $\bar{M}=\{p \in M ; \bar{S}(p) \neq 0\}$. Since $M$ is a ruled hypersurface, the sectional curvature $\bar{K}$ at points of $\bar{M}$ is not identically zero. It follows from Lemma 2.4 applied to $\bar{M}$ that at each point of the image of the Gauss map the first normal space $N_{1}$ has dimension $\leq 1$. We have $\bar{M}=\bar{M}_{0} \cup \bar{M}_{1}$, where at $\bar{M}_{0}$ the Gauss map is totally geodesic in $S^{n}$ and $\bar{M}_{1}$ is the open subset of points where $N_{1}$ has dimension 1 .

Let $V_{1}$ be a connected component of $\bar{M}_{1}$, let $X: U \subset \Lambda \rightarrow V_{1} \subset R^{n+1}$ be a Gauss parametrization and let $g: L^{2} \rightarrow S^{n}$ be the associated local parametrization of the Gauss map of $V_{1}$. It follows from Proposition 2.1 that there exists a totally geodesic submanifold $S^{3} \subset S^{n}$ such that $g\left(L^{2}\right) \subset S^{3}$ is a ruled Weingarten surface which satisfies $H^{2}+c^{2}(K-1)=0$. Moreover, $V_{1}$ is contained in a euclidean product $Q^{3} \times \mathbf{R}^{n-3}$, where $Q^{3} \subset \mathbf{R}^{4}$ is a ruled Weingarten surface with constant index of relative nullity $\nu=1$.

Using Proposition 2.2, we obtain that either $g$ is a minimal immersion in $S^{3}$ or $K=0, H=c$, and the image of $g$ is contained in the product of two circles of $S^{3}$. It follows from Proposition 2.3, that $Q^{3}$ is an open subset of a cone over the image of $g$, i.e. $V_{1}$ satisfies (ii).

Let $V_{0}$ be a connected open subset of $\bar{M}_{0}$. We have a Gauss parametrization for $V_{0}$ and $g$ the associated local parametrization of the image of the Gauss map of $V_{0}$. Since $g$ is totally geodesic in $S^{n}$, the normal bundle $\Lambda$ of the immersion $g$ is parallel in $\mathbf{R}^{n+1}$. Hence, using the Gauss parametrization we obtain that $V_{0}$ is an open subset of $Q^{2} \times \mathbf{R}^{n-2}$, where $Q^{2} \subset \mathbf{R}^{3}$ is a ruled Weingarten surface. It follows from the classical result of Beltrami [B] and Dini [D] that $Q^{2}$ is a ruled helicoidal surface or a hyperboloid of revolution, i.e. $V_{0}$ satisfies (iii).

We now observe that the boundary of $V_{0}$ does not intersect the boundary of $V_{1}$, since the determinant of the second fundamental form of the image of the Gauss map of $V_{1}$ in $S^{3}$ is bounded away from zero. Moreover, the boundaries of $V_{0}$ and of $V_{1}$ do not contain points where the scalar curvature $\bar{S}$ is zero. Since $M$ is connected, this concludes the proof of the theorem. Q.E.D.

Remark 2.28. The ruled Weingarten surfaces $Q^{2} \subset \mathbf{R}^{3}$ classified by Beltrami and Dini are given by

$$
X(s, t)=\left(a \cos s+c t \sin s, a \sin s-c t \cos s, b s+\sqrt{1-c^{2}} t\right)
$$

where $a, b, c$ are constants. 
Proof of Corollary C. We use Theorem B. If $M$ is complete, then it cannot be a cone. If $M$ splits as in (iii), then $M=Q^{2} \times \mathbf{R}^{2}$, where $Q^{2}$ is a complete ruled helicoidal surface or a hyperboloid of revolution. If $M$ is flat, it follows from [HN] that $M$ is a cylinder over a complete curve. Q.E.D.

Proof of Theorem A. If $M^{n} \subset \mathbf{R}^{n+1}, n \geq 4$, is a complete hypersurface, it follows from $[\mathrm{DG}]_{2}$ that $M$ is rigid, unless it contains an open subset $U$ which is completely ruled.

We will show that the existence of such a subset $U$ contradicts the hypothesis of Theorem A. In fact, if we apply Theorem B to each connected component $U_{0}$ of $U$, we conclude that $U_{0}$ is completely ruled and flat. We consider a connected component of $U_{0}$ where the nullity is $n-1$. Then the ruling coincides with the nullity and therefore the nullity is complete. The argument used in [HN] implies that this component of $U_{0}$ is a cylinder over a curve (not necessarily complete). Moreover, each connected component of $U_{0}$ where the nullity is $n$ is totally geodesic. Hence, in both cases we obtain open subsets of type $L^{3} \times \mathbf{R}^{n-3}$, with $L^{3}$ unbounded, which is a contradiction. Therefore, $M$ is rigid. Q.E.D.

\section{REFERENCES}

[B] E. Beltrami, Risoluzione di un problema relativo alla teoria delle superficie gobbe, Ann. Mat. Pura Appl. 7 (1865), 139-150.

[BDJ] L. Barbosa, M. Dajczer and L. Jorge, Minimal ruled submanifolds in spaces of constant curvature, Indiana Univ. Math. J. 33 (1984), 531-547.

[C] E. Cartan, La deformation des hypersurfaces dans l'espace euclidien réel e $n$ dimensions, Bull. Soc. Math. France 44 (1916), 65-99.

[D] O. Dini, Sulle superficie gobbe nele quali uno dei due raggi di curvatura principale è una funzione dell'altro, Ann. Mat. Pura Appl. 7 (1865), 205-210.

[DG 1 ] M. Dajczer and D. Gromoll, Gauss parametrizations and rigidity aspects of submanfolds, J. Differential Geometry 22 (1985), 1-12.

[DG 2 ] _ Rigidity of complete, Euclidean hypersurfaces, Preprint.

[HN] P. Hartman and L. Nirenberg, On spherical image maps whose Jacobians do not change sign, Amer. J. Math. 81 (1959), 901-920.

[L] B. Lawson, Complete minimal surfaces in $S^{3}$, Ann. of Math. 92 (1970), 335-374.

[S] U. Sbrana, Sulle varietà ad $n-1$ dimensione deformabili nello spazio euclideo ad $n$ dimensione, Rend. Circ. Mat. Palermo 27 (1909), 1-45.

[Sp] M. Spivak, A comprehensive introduction to differential geometry, Publish or Perish, 1979.

IMPA, Estrada Dona Castorina 110, Jardim Botânico, 22.460 Rio de Janeiro-RJ, Brasil

Departamento de Matemática, Universidade de BrasiĹia, 70.910 BrasiĹia-DF, Brasil 